\title{
Stabilizability over Deterministic Relay Networks
}

\author{
Miroslav Pajic
}

\author{
Shreyas Sundaram
}

\author{
George J. Pappas
}

\begin{abstract}
We consider the problem of linear system stabilization using a set of decentralized controllers that communicate with the plant's sensors over a network that employs linear network coding. Our analysis is built upon an existing algebraic description of deterministic relay networks, which is able to model broadcast transmissions and multiple access channel constraints. Since these networks can be described as linear time-invariant systems with specific transfer functions, this network representation allows us to reason about the control system and network (and their interaction) using a common mathematical framework. In this paper we characterize algebraic and topological stabilizability conditions for a wide class of these networks. Our analysis shows that the (algebraic) structure of a network required for stabilization of a dynamical plant can be related to the plant's dynamics; in particular, we prove that the geometric multiplicities of the plant's unstable eigenvalues play a key role in the ability to stabilize the system over such networks.
\end{abstract}

\section{INTRODUCTION}

Networked Control Systems (NCS) theory focuses on the design and analysis of systems where communication networks are used as interfaces between the physical processes (i.e., plants) and controllers [1], [2]. In these systems, a set of distributed sensors and actuators is connected to a realtime network used to deliver information from the sensors to the controllers and actuators. Despite the inherently tight coupling between the network and physical dynamics, the designs of the network and controllers are typically very loosely connected, often resulting in over-constrained network design specifications.

For example, in most systems the design goal for the communication networks is to deliver information generated at source nodes (i.e., all plant sensors) to a set of intended destination nodes (i.e., controllers). To illustrate this, consider the idealized network (without delays on any of the links) shown in Fig. 1. Here, $p$ plant sensors transmit data over the network to $m$ actuators that apply inputs to the plant computed by local controllers (located at the actuators). Each sensor injects one unit ${ }^{1}$ of information per time-step into

This research has been partially supported by the NSF-CNS 0931239 award, by a grant from NSERC, and by the TerraSwarm Research Center, one of six centers supported by the STARnet phase of the Focus Center Research Program (FCRP) a Semiconductor Research Corporation program sponsored by MARCO and DARPA.

M. Pajic and G. J. Pappas are with the Department of Electrical and Systems Engineering, University of Pennsylvania, Philadelphia, PA, USA 19104. Email: \{pajic, pappasg\}eseas.upenn.edu. S. Sundaram is with the Department of Electrical and Computer Engineering, University of Waterloo, Waterloo, ON, Canada, N2L 3G1. Email: ssundara@uwaterloo.ca.

${ }^{1}$ We focus on the case where real-valued measurements are transmitted over the network, although in practice, the measurements and the implemented control computations are quantized to some finite, but sufficiently high, precision. the network. If the objective is to fully recover all of these values at every actuator at each time-step, the network needs a capacity of $p$ units per time-step.

On the other hand, if the objective is not to transmit all of the source information but rather to stabilize the system over this network, a network with low capacity will not necessarily be an impediment. Consequently, it is necessary to provide a set of network conditions under which a given linear system can be stabilized. In a recent paper [3], we started to address this problem within the Wireless Control Networks (WCN) architecture, in which the network itself acts as a linear dynamical compensator [4]. In this work, we consider a more general network model and provide algebraic and topological stabilizability conditions for a wide class of networks that employ linear network coding (LNC); we will refer to these networks as LNC networks. We show that the (algebraic) structure of an LNC network required for stabilization of a dynamical plant can be related to the plant's dynamics; in particular, we show that the geometric multiplicity of the plant's unstable eigenvalues plays a key role. This supplements other recent investigations of the effect of the plant's eigenvalues on the network conditions required for stabilization, including [3] where wireless control networks were studied, and [5], where a minimum rate of transmission between controllers and actuators was derived based on the eigenstructure of the plant. We build upon the algebraic description of deterministic relay networks provided in [6], which was used to show a connection between algebraic network coding and a deterministic network model referred to as ADT [7]. As shown in [6], the algebraic framework is able to capture the properties of ADT models, which include support for modeling of broadcast transmissions, interference and multiple access channel (MAC) constraints.

The fact that relay nodes in LNC networks can process and mix incoming data (by applying appropriate linear functions) instead of just forwarding it without any changes can be captured by a very simple algebraic description [8]. The use of algebraic methods to model LNC networks as a whole, which was introduced in [8] and extended in [6], [9], has paved the way for the application of algebraic methods to their analysis. Since these networks can be modeled as linear time-invariant (LTI) systems with specific transfer functions, this network representation has enabled reasoning about the control system and network (and their interaction) using a common mathematical framework. In addition, [10] provided intuition into the existence of decentralized fixed modes (defined in [11], [12]) from a network coding perspective.

This paper is organized as follows. In Section II, we describe the problem considered in the paper, along with 


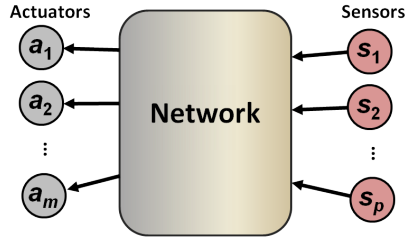

Fig. 1. Communication network used to transmit plant measurements from a set of $p$ plant's sensors to the $m$ actuators.

the system model that includes the algebraic formulation of the ADT network model. In Section III, we present the main result of the paper, a set of sufficient algebraic (and for some networks, topological) network conditions that ensure the existence of a set of stabilizing controllers (placed at the actuators). Section IV reviews the concept of decentralized fixed modes, which is then used in Section $\mathrm{V}$ to prove the network conditions. Finally, in Section VI we give some concluding remarks.

\section{A. Notation and Terminology}

We use $\mathbf{e}_{i}$ to denote the column vector (of appropriate size) with a 1 in its $i$-th position and 0's elsewhere, and $\mathbf{A}^{\prime}$ indicates the transpose of matrix $\mathbf{A}$. For a matrix $\mathbf{T}$ we denote the $(i, j)^{t h}$ element by $t_{i j}$. For a square matrix $\mathbf{M}, \Lambda(\mathbf{M})$ denotes the set of eigenvalues of $\mathbf{M}$. The cardinality of a set $\mathcal{S}$ is denoted by $|\mathcal{S}|$, while $\mathcal{S}^{\complement}$ specifies the complement set of $\mathcal{S}$. For two sets $\mathcal{S}$ and $\mathcal{R}$, we use $\mathcal{S} \backslash \mathcal{R}$ to denote the set of elements in $\mathcal{S}$ that are not in $\mathcal{R}$. Finally, we define the sets $\mathcal{M}=\{1,2, \ldots, m\}$ and $\mathcal{P}=\{1,2, \ldots, p\}$.

\section{PRoblem Description}

We consider the setup from Fig. 2, where a system (i.e., plant) is to be controlled by a set of dynamical controllers, with a single controller being located at each actuator. ${ }^{2}$ A communication network is used to deliver information (i.e., sensor measurements) from the sensors to controllers (i.e., actuators). In this work we focus on detectable and stabilizable LTI systems described by:

$$
\begin{aligned}
\mathbf{x}[k+1] & =\mathbf{A} \mathbf{x}[k]+\mathbf{B u}[k] \\
\mathbf{y}[k] & =\mathbf{C x}[k],
\end{aligned}
$$

where $\mathbf{x}[k] \in \mathbb{R}^{n}, \mathbf{u}[k] \in \mathbb{R}^{m}$, and $\mathbf{y}[k] \in \mathbb{R}^{p}$ denote the state of the system, its input and output vectors, respectively. We will refer to this system as $\Sigma=\{\mathbf{A}, \mathbf{B}, \mathbf{C}\}$. We assume that the measurements of the output vector $\mathbf{y}[k]=$ $\left[y_{1}[k] y_{2}[k] \ldots y_{p}[k]\right]^{T}$ are being provided by the sensors from the set $\mathcal{S}=\left\{s_{1}, s_{2}, \ldots, s_{p}\right\}$. In addition, the input vector signals $\mathbf{u}[k]=\left[u_{1}[k] u_{2}[k] \ldots u_{m}[k]\right]^{T}$ are being applied to the plant by actuators from the set $\mathcal{A}=\left\{a_{1}, a_{2}, \ldots, a_{m}\right\}$.

\section{A. Network Model}

The network model used in this work is based on the model from [6]. The network is represented by a directed graph $\mathcal{G}=(\mathcal{V}, \mathcal{E})$ with a node set $\mathcal{V}$ and an edge set $\mathcal{E}$. Each

\footnotetext{
${ }^{2}$ It was shown in [13] that the optimal location for controllers in NCS with packet drops is at the plant actuators.
}

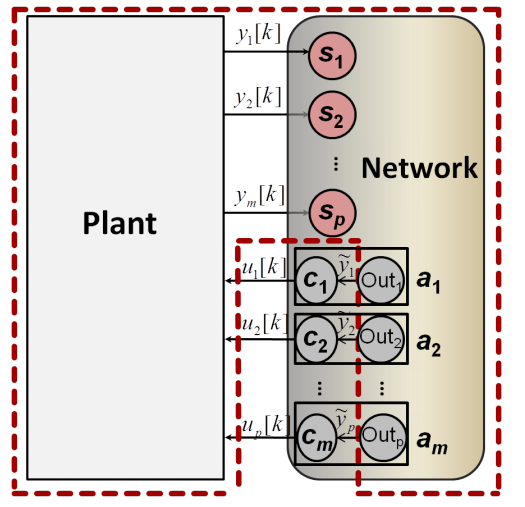

Fig. 2. Networked Control System where a network is used to deliver information from the plant's sensors $s_{1 . . p}$ to controllers located at the actuators $a_{1 . . m}$. Each actuator $a_{i}$ consists of network port $O u t_{i}$ and controller $c_{i}$ that provides plant input $u_{i}$. The red line highlights a new dynamical system, composed of the initial plant and communication network.

node $v \in \mathcal{V}$ consists of input ports $\operatorname{In}(v)$ and output ports $\operatorname{Out}(v)$, and for any edge $\left(e_{1}, e_{2}\right) \in \mathcal{E}, e_{1} \in O u t\left(v_{i}\right)$ and $e_{2} \in \operatorname{In}\left(v_{j}\right)$ for some $v_{i}, v_{j} \in \mathcal{V}$ (i.e., each edge connects an output port of a node to another node's input port). ${ }^{3}$ We use $\mathcal{E}\left(v_{i}, v_{j}\right)$ to denote the set of edges from node $v_{i}$ to node $v_{j}$.

In this setup, the set of sensors $\mathcal{S} \subset \mathcal{V}$ is the set of information sources, while the set of actuators $\mathcal{A} \subset \mathcal{V}$ is effectively the set of destinations. Thus, each sensor $s_{j}$ has a single input port (in the set $\operatorname{In}\left(s_{j}\right)$ ), and the port is dedicated to the sequence $y_{j}[k]$ (i.e., the $j^{t h}$ plant's output). In addition, we assume that each sensor node has at least one output port - i.e., for each sensor $s_{j},\left|O u t\left(s_{j}\right)\right| \geq 1$. Similarly, each actuator $a_{i}$ has a single output port (in the set $\operatorname{Out}\left(a_{i}\right)$ ) dedicated to input $\tilde{Y}_{i}$ into the controller located at the actuator (see Fig. 3).

The network employs linear network coding over reals (i.e., field $\mathbb{R}$ ) to transmit information from sources to destinations. ${ }^{4}$ We do not restrict the model to acyclic networks; we consider networks that potentially contain cycles, and where some links may introduce delays that are equal to the sampling period of the plant. Accordingly, the derived network conditions for system stabilization can also be used for the case with an acyclic, delay-free network. ${ }^{5}$

1) Algebraic Representation of the Network: For any node $v$, each output port $e \in O u t(v)$ transmits one real number per time unit. In particular, each input and output port $e$ has an associated sequence of values $X(e, k)$, where $k \in \mathbb{N}$. We will denote the power series for this port by $X(e)=$ $\sum_{k=0}^{\infty} X(e, k) z^{k}$, where $z$ represents a unit delay. ${ }^{6}$ For $e \in$ $\operatorname{In}(v)$, where $v \in \mathcal{V} \backslash \mathcal{S}$,

$$
X(e)=\sum_{e_{i} \text { s.t. }\left(e_{i}, e\right) \in \mathcal{E}} X\left(e_{i}\right),
$$

${ }^{3}$ Note that we use the notation from [6]. In [9], input and output ports are defined from a channel-centric perspective.

${ }^{4}$ In practice, quantized values with a sufficiently large number of bits are transmitted over communication channels [5].

${ }^{5}$ This scenario, although idealized, can be used to model networks with delays that are significantly smaller than the plant's sampling period.

${ }^{6}$ In general, we should denote this term as $X(e, z)$. However, to simplify the notation we have opted to use the former representation. 


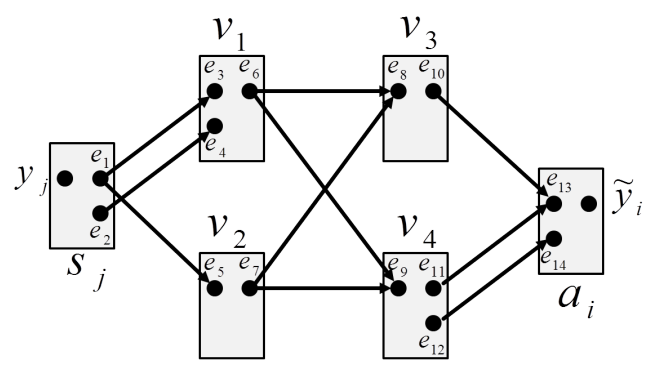

Fig. 3. Example network from [14].

which models transmissions incoming to the input port $e$. Note that this representation allows for modeling MAC constraints.

Furthermore, for $e \in O u t(v)$, where $v \in \mathcal{V}$,

$$
X(e)=\sum_{e_{i} \in \operatorname{In}(v)} \gamma\left(e_{i}, e\right) X\left(e_{i}\right),
$$

which describes an LTI function implemented by each node. Similarly, for $e \in \operatorname{In}\left(s_{j}\right)_{\tilde{Y}}$ we have that $X(e)=Y_{j}$, and for $e \in \operatorname{Out}\left(a_{i}\right), X(e)=\tilde{Y}_{i}$. As in [8], [6], [9], we assume that the each node acts as an LTI system, and thus here, $\gamma$ are either constants or real rational functions of the delay parameter $z$. Finally, we use $\Gamma$ to denote the set of all free parameters used to specify these linear functions.

To illustrate this consider the network from Fig. 3. Here, $X\left(e_{1}\right)=\gamma\left(y_{j}, e_{1}\right) Y_{j}$ and $X\left(e_{2}\right)=\gamma\left(y_{j}, e_{2}\right) Y_{j}$ for output ports of the sensor node $s_{j}$, and $X\left(e_{6}\right)=\gamma\left(e_{3}, e_{6}\right) X\left(e_{3}\right)+$ $\gamma\left(e_{4}, e_{6}\right) X\left(e_{4}\right)$ for the output port of node $v_{1}$. In addition, $X\left(e_{4}\right)=X\left(e_{2}\right)$. However, $X\left(e_{3}\right)=X\left(e_{5}\right)=X\left(e_{1}\right)$ since there are two edges from the output port $e_{1}$ of node $s_{j}$ to input ports $e_{3}$ and $e_{5}$ of nodes $v_{1}$ and $v_{2}$, respectively. This effectively specifies a broadcast constraint that values sent over the edges are the same. Finally, $X\left(e_{8}\right)=X\left(e_{9}\right)=$ $X\left(e_{6}\right)+X\left(\tilde{\sim}_{7}\right)$ describes a MAC constraint.

We use $\tilde{\mathbf{Y}}_{I n\left(a_{i}\right)}$, for $i \in \mathcal{M}$, to denote the vector containing data from all input ports of the actuator $a_{i}$. We

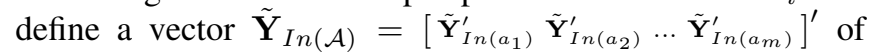
size $n_{\tilde{\mathbf{Y}}}$, and we introduce a binary $m \times n_{\tilde{\mathbf{Y}}}$ matrix $\mathbf{T}$ where each element $t_{i k}=1$ if and only if the $k^{t h}$ element of the vector $\tilde{\mathbf{Y}}_{\operatorname{In}(\mathcal{A})}$ corresponds to an input port in actuator $a_{i}$. Similarly, by $\mathbf{Y}_{\operatorname{In}\left(s_{j}\right)}$, for $j \in \mathcal{P}$, we denote the vector containing data from the input port of the node $s_{j}$, while

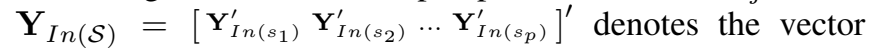
corresponding to sensor measurements.

For the above network model, a transfer function matrix $\mathbf{G}_{\operatorname{In}(\mathcal{S}), \operatorname{In}(\mathcal{A})}(z)$ can be obtained such that $\tilde{\mathbf{Y}}_{\operatorname{In}(\mathcal{A})}(z)=$ $\mathbf{G}_{\operatorname{In}(\mathcal{S}), \operatorname{In}(\mathcal{A})}(z) \mathbf{Y}_{\operatorname{In}(\mathcal{S})}(z)$ (e.g., see [6], [8], [9]). Thus, for each set $I \subseteq \mathcal{M}$ and $J \subseteq \mathcal{P}$, we use $\mathbf{G}_{\operatorname{In}\left(\mathcal{S}_{J}\right), \operatorname{In}\left(\mathcal{A}_{I}\right)}(z)$ to denote the transfer function matrix from the input ports of all sensors from the set $\mathcal{S}$ with indices in the set $J$, to the input ports of all actuator nodes from $\mathcal{A}$ with indices in the set $I$.

\section{B. Problem Formulation}

In this paper we address the following question: What conditions should the network satisfy such that there exist network parameters (from $\Gamma$ ) for which we can derive a set of stabilizing dynamical compensators located at the plant's actuators? Note that we will derive these conditions using the rank of network transfer functions. As we will show in Section III, for some networks (e.g., wired networks with point-to-point links) these conditions can be easily mapped into topological conditions imposed on the graph $\mathcal{G}$. This will complement and extend existing capacity-oriented network conditions for unicast/multicast communication over LNC networks (e.g., [6], [9], [8]), to the setting of stabilizing a given plant over such networks.

\section{MAIN RESULT}

To introduce our results we will use the following notation. For sets $I=\left\{i_{1}, i_{2}, \ldots, i_{|I|}\right\} \subseteq \mathcal{M}$ and $J=\left\{j_{1}, \ldots, j_{|J|}\right\} \subseteq$ $\mathcal{P}$ we define the matrices $\mathbf{B}_{I}=\left[\begin{array}{llll}\mathbf{b}_{i_{1}} & \mathbf{b}_{i_{2}} & \ldots & \mathbf{b}_{i_{|I|}}\end{array}\right]$ and $\mathbf{C}_{J}=\left[\begin{array}{llll}\mathbf{c}_{j_{1}}^{\prime} & \mathbf{c}_{j_{2}}^{\prime} & \ldots & \mathbf{c}_{j_{|J|} \mid}^{\prime}\end{array}\right]^{\prime}$, where $\mathbf{b}_{i}$ denotes the $i^{t h}$ column of matrix $\mathbf{B}$, while $\mathbf{c}_{j}$ is the $j^{\text {th }}$ row of matrix $\mathbf{C}$. The algebraic representation of the network as an LTI system (from Section II) allows us to prove the following result.

Theorem 1: Let $\lambda$ be an unstable eigenvalue of $\mathbf{A}$ and for each subset $I \subseteq \mathcal{M}$, let $d_{I}=n-\operatorname{rank}\left[\begin{array}{ll}\mathbf{A}-\lambda \mathbf{I} & \mathbf{B}_{I}\end{array}\right]$. Suppose that for each subset $I \subseteq \mathcal{M}$ there exists a subset $F \subseteq \mathcal{P}$ with $d_{I}$ plant outputs such that the following hold

$$
\begin{gathered}
\operatorname{rank}\left[\begin{array}{cc}
\mathbf{A}-\lambda \mathbf{I} & \mathbf{B}_{I} \\
\mathbf{C}_{F} & \mathbf{0}
\end{array}\right]=n, \\
\max _{\Gamma} \operatorname{rank}\left(\mathbf{G}_{I n\left(\mathcal{S}_{F}\right), \operatorname{In}\left(\mathcal{A}_{\mathcal{M} \backslash I}\right)}(z)\right)=d_{I} .
\end{gathered}
$$

Furthermore, suppose the above holds true for all unstable eigenvalues $\lambda$ of $\mathbf{A}$. Then there exists an assignment of the parameters from $\Gamma$ for which the system can be stabilized by the controllers assigned to the actuators.

We will prove this result in Section V. The above theorem requires that we examine this condition for all unstable eigenvalues of $\mathbf{A}$. However, it is possible to provide a simpler sufficient condition by noting that for any unstable eigenvalue $\lambda$ of $\mathbf{A}, \operatorname{rank}(\mathbf{A}-\lambda \mathbf{I}) \geq n-d$, where $d$ denotes the largest geometric multiplicity of any unstable eigenvalue of A. Consider any unstable eigenvalue $\lambda$ of $\mathbf{A}$, and any set $I \subset \mathcal{M}$ with the corresponding $d_{I}$. From the definition of $d$ it holds that $d \geq d_{I}$. Denote with $F$ a subset of $\mathcal{P}$ with $d_{I}$ elements that satisfies (2). If for all $i \in \mathcal{M}$ and $j \in \mathcal{P}$,

$$
\max _{\Gamma} \operatorname{rank}\left(\mathbf{G}_{O u t\left(s_{j}\right), \operatorname{In}\left(a_{i}\right)}(z)\right) \geq d,
$$

since $\operatorname{Out}\left(s_{j}\right) \subseteq \operatorname{Out}\left(\mathcal{S}_{F}\right)$ and $\operatorname{In}\left(a_{i}\right) \subseteq \operatorname{In}\left(\mathcal{A}_{\mathcal{M} \backslash I}\right), \forall j \in$ $F, i \in \mathcal{M} \backslash I$, we have $\max _{\Gamma} \operatorname{rank}\left(\mathbf{G}_{\text {Out }\left(\mathcal{S}_{F}\right), \operatorname{In}\left(\mathcal{A}_{\mathcal{M} \backslash I}\right)}(z)\right) \geq$ $d$. Thus, since $\left|\mathcal{S}_{F}\right|=d_{I} \leq d$ and $\forall e \in \operatorname{Out}\left(s_{j}\right), \gamma\left(s_{j}, e\right)$ is a free parameter, using the same approach as in the proof of Theorem 1 we can show that (3) is satisfied if (4) is true. The latter can be also specified using the minimum value of all $s_{j}-a_{i}$ cuts in the network, i.e., $\operatorname{mincut}\left(s_{j}, a_{i}\right)$.

Definition 1 ([7], [6]): A $s_{j}-a_{i}$ cut $\Theta$ is a partition of the set of nodes $\mathcal{V}$ into two disjoint sets $\Theta$ and $\Theta^{C}$, where $s_{j} \in \Theta$ and $a_{i} \in \Theta^{\complement}$. For any cut $\Theta, \mathcal{G}_{\Theta}$ is the incidence matrix for the bipartite graph with ports in $\Theta$ and $\Theta^{\complement}$. Then,

$$
\operatorname{mincut}\left(s_{j}, a_{i}\right)=\min _{\Theta} \operatorname{rank}\left(\mathcal{G}_{\Theta}\right)
$$


From [6], if $\operatorname{mincut}\left(s_{j}, a_{i}\right) \geq d$ then the condition from (4) is satisfied, which allows us to obtain the corollary below.

Corollary 1: For a detectable and stabilizable system $\Sigma=$ $(\mathbf{A}, \mathbf{B}, \mathbf{C})$ let $d$ denote the largest geometric multiplicity of any unstable eigenvalue of $\mathbf{A}$. If for every sensor $s_{j} \in \mathcal{S}$ and actuator $a_{i} \in \mathcal{A}$, mincut $\left(s_{j}, a_{i}\right) \geq d$, then the system can be stabilized using a dynamic controller at each actuator.

\section{A. Topological Conditions for System Stabilization}

Corollary 1 provides an important algebraic condition for the existence of network parameters and a stabilizing set of controllers at the actuators. However, in the general case, the algebraic cut value from Definition 1 might not be equal to the graph-theoretic min cut (i.e., the smallest total capacity on edges from one set of vertices to its complement). This effectively prevents direct mapping of the corollary into a topological condition for the graph $\mathcal{G}$. For example, the mincut value (i.e., $\operatorname{mincut}\left(s_{j}, a_{i}\right)$ ) of the network from Fig. 3 is equal to one (for the cut $\Theta=\left\{s_{j}, v_{1}, v_{2}\right\}$ ). This is caused by the fact that the model allows us to incorporate MAC and broadcast constraints. Thus, some of the network edges become linearly dependent, as we have seen in the example. As shown in [6], [14], this may reduce the rank of a cut.

A polynomial-time algorithm to compute the mincut value was introduced in [14]. To the best of our knowledge, there does not exist a quantitative dependency between the topology of a network and the algebraic min-cut. On the other hand, for networks where all the links are linearly independent, mapping the min-cut condition into topological requirements is straightforward. For example, in the wired (or point-to-point) LNC networks described in [8] (which can also be mapped into the model from Section II), all links are linearly independent. Thus, for these networks the min-cut is equal to the network's edge-connectivity.

It is worth noting here that for almost any plant $\Sigma=$ $\{\mathbf{A}, \mathbf{B}, \mathbf{C}\}$ (i.e., for those except a set of measure zero), the maximal geometric multiplicity of any nonzero eigenvalue is equal to one, meaning that $d$ from Corollary 1 is also equal to one [15], [3]. Therefore, from Corollary 1 the network has to provide a min-cut of size one between any sensor and any actuator. In this case, if the initial graph $\mathcal{G}$ is connected (in which case, linearly dependent edges cannot reduce rank to less than one) it is possible to stabilize the plant using the network and a set of controllers located at actuators.

In the rest of this paper we will provide a proof of Theorem 1. The proof utilizes the fact that we can consider the plant and communication network as a single dynamical system, which allows us to frame the problem as stabilization using a decentralized set of controllers. Accordingly, before we proceed with the proof, in the next section we review the notion of fixed modes from decentralized control theory.

\section{Decentralized FiXed Modes}

The problem of decentralized control, where each of the controllers cannot observe the full state of the system, can be formulated as a static output feedback problem where a set of structural constraints has been imposed on the feedback matrix [11], [12]. To reason about stabilization of such systems, Wang and Davison introduced in [11] the concept of fixed modes - eigenvalues of the plant that are fixed (i.e., cannot be moved) despite any choice for the feedback gains.

To formally define the notion of fixed modes we consider a setup where a discrete-time system $\Sigma=\{\mathbf{A}, \mathbf{B}, \mathbf{C}\}$ is to be controlled by a set of $m$ dynamical controllers. Each input $u_{i}, i \in \mathcal{M}$, is provided by a dynamical compensator that can use measurements from only a subset of the plant's sensors. These feedback constraints can be specified as follows.

Definition 2: The decentralized feedback structure constraints are specified by the binary matrix $\overline{\mathbf{F}} \in\{0,1\}^{m \times p}$, where for all $i \in \mathcal{M}$ and $j \in \mathcal{P}, \bar{f}_{i, j}=1$ if and only if output $y_{j}$ can be used to calculate input $u_{i}$. Furthermore, the decentralized feedback pattern sets $J_{1}, J_{2}, \ldots, J_{m} \subseteq \mathcal{P}$ are defined as $J_{i}=\left\{j \mid \bar{f}_{i j}=1, j \in \mathcal{P}\right\}$.

From the above definition, $\bar{f}_{i j}=1$ and $j \in J_{i}$ if and only if there exist a feedback link between the sensor $s_{j}$ and actuator $a_{i}$. Thus, we can describe the decentralized LTI controllers at each actuator as $(i=1, \ldots, m)$

$$
\begin{aligned}
\mathbf{z}_{i}[k+1] & =\mathbf{F}_{i} \mathbf{z}_{i}[k]+\sum_{j \in J_{i}} \mathbf{q}_{i j} y_{j}[k] \\
u_{i}[k] & =\mathbf{h}_{i}^{\prime} \mathbf{z}_{i}[k]+\sum_{j \in J_{i}} k_{i j} y_{j}[k] .
\end{aligned}
$$

Here, $\mathbf{z}_{i} \in \mathbb{R}^{n_{i}}$ is the controller's state vector, while matrix $\mathbf{F}_{i}$ and vectors $\mathbf{q}_{i}, \mathbf{h}_{i}$ are of appropriate dimensions.

To introduce fixed modes and stabilizability conditions, we start by defining the set of output feedback matrices based on the decentralized feedback structure:

$$
\mathbf{K}_{f}=\left\{\mathbf{K} \in \mathbb{R}^{m \times p} \mid k_{i j}=0 \text { if } \bar{f}_{i j}=0\right\} .
$$

Definition 3 ([11], [12]): For the system $\Sigma=$ $\{\mathbf{A}, \mathbf{B}, \mathbf{C}\}$, the set $\Lambda_{f}=\bigcap_{\mathbf{K} \in \mathbf{K}_{f}} \Lambda(\mathbf{A}+\mathbf{B K C})$ is called the set of fixed modes with respect to the feedback structure constraints specified by the matrix $\overline{\mathbf{F}}$.

Therefore, the fixed modes are the eigenvalues of A + BKC that cannot be moved by static output feedback, regardless of the utilized matrix $\mathbf{K} \in \mathbf{K}_{f}$. Fixed modes play a crucial role in the stabilizability analysis of decentralized control systems due to the following theorem.

Theorem 2 ([11], [12]): The system $\Sigma=\{\mathbf{A}, \mathbf{B}, \mathbf{C}\}$ can be stabilized using the set of controllers defined in (6) if and only if all of the system's fixed modes are stable.

To provide an algebraic characterization for the fixed modes of a decentralized control system, for any subset $I \subseteq \mathcal{M}$ we define the set

$$
J=\bigcup_{i \in \mathcal{M} \backslash I} J_{i},
$$

containing the indices of all sensors whose measurements are used by at least one controller with index in the set $\mathcal{M} \backslash I$.

Theorem 3 ([16]): A complex number $\lambda$ is a fixed mode of the system $\Sigma=\{\mathbf{A}, \mathbf{B}, \mathbf{C}\}$ from (1) if and only if there exists a subset $I \subseteq \mathcal{M}$ such that, for $J$ defined in (8),

$$
\operatorname{rank}\left[\begin{array}{cc}
\mathbf{A}-\lambda \mathbf{I} & \mathbf{B}_{I} \\
\mathbf{C}_{J} & \mathbf{0}
\end{array}\right]<n .
$$


There are a variety of other algebraic tests for fixed modes with respect to a given feedback structure (e.g., [16], [17]). Furthermore, to facilitate analysis of large systems with uncertain parameters and feedback structural constraints, the authors in [12] introduced graph-theoretic characterizations of fixed modes, which we exploited in [3] to obtain generic topological conditions for control over Wireless Control Networks. In the next section, we will use the characterization from Theorem 3 to prove our main result.

\section{Algebraic Conditions for System STABILIZATION OVER LNC NETWORKS}

In this section we provide a proof of Theorem 1 . We use a similar approach as in [3], where we considered the plant and network as a new dynamical system, as shown in Fig. 2. We start by obtaining a state-space realization for the transfer function matrix $\mathbf{G}_{\operatorname{In}(\mathcal{S}), \operatorname{In}(\mathcal{A})}(z)$ (defined in Section II), specified by matrices $\mathbf{A}^{\text {net }}, \mathbf{B}^{\text {net }}, \mathbf{C}^{\text {net }}, \mathbf{D}^{\text {net }}$. We can then describe the network with a state $\mathbf{w}[k] \in \mathbb{R}^{N}$ (where $N$ is the size of $w$ ) and dynamics

$$
\begin{aligned}
\mathbf{w}[k+1] & =\mathbf{A}^{n e t} \mathbf{w}[k]+\mathbf{B}^{n e t} \mathbf{y}[k] \\
\tilde{\mathbf{y}}_{I n(\mathcal{A})}[k] & =\mathbf{C}^{n e t} \mathbf{w}[k]+\mathbf{D}^{n e t} \mathbf{y}[k] .
\end{aligned}
$$

If we assume for now that the controllers at actuators do not provide inputs to the plant, we can define a new system $\tilde{\Sigma}$ comprised of the plant and network. The system $\tilde{\Sigma}$, with state $\tilde{\mathbf{x}}[k]=\left[\begin{array}{c}\mathbf{x}[k] \\ \mathbf{w}[k]\end{array}\right]$, evolves as:

$$
\begin{aligned}
\tilde{\mathbf{x}}[k+1] & =\underbrace{\left[\begin{array}{cc}
\mathbf{A} & \mathbf{0} \\
\mathbf{B}^{n e t} \mathbf{C} & \mathbf{A}^{n e t}
\end{array}\right]}_{\tilde{\mathbf{A}}}\left[\begin{array}{c}
\mathbf{x}[k] \\
\mathbf{w}[k]
\end{array}\right]+\underbrace{\left[\begin{array}{c}
\mathbf{B} \\
\mathbf{0}
\end{array}\right]}_{\tilde{\mathbf{B}}} \mathbf{u}[k], \\
\tilde{\mathbf{y}}_{I n(\mathcal{A})}[k] & =\underbrace{\left[\begin{array}{ll}
\mathbf{D}^{n e t} \mathbf{C} & \mathbf{C}^{n e t}
\end{array}\right]}_{\tilde{\mathbf{C}}}\left[\begin{array}{c}
\mathbf{x}[k] \\
\mathbf{w}[k]
\end{array}\right],
\end{aligned}
$$

and the output of the new system $\tilde{\Sigma}=\{\tilde{\mathbf{A}}, \tilde{\mathbf{B}}, \tilde{\mathbf{C}}\}$ at each time-step $k$ is the data sent by the network to the controllers stationed at the actuators.

From (11) it follows that the problem of controlling the initial system over the available network can be mapped into control of the new system $\tilde{\Sigma}$ using a set of $m$ decentralized controllers (stationed at actuators), with feedback constraints imposed by the matrix T. It is worth noting that the algebraic representation of the network behavior has enabled us to consider the plant and network as a new linear system. This in turn has allowed for the formulation of our stabilization problem within the decentralized control framework.

To derive network conditions for which there exists a set of stabilizing controllers at the actuators, we provide conditions for which the system $\tilde{\Sigma}$ from (11) does not have unstable fixed modes for feedback structural constraints specified by the matrix $\mathbf{T}$. Our goal is to use the algebraic condition from Theorem 3 to achieve this. However, in this case it is necessary to derive an equivalent definition of the set $J$

\footnotetext{
${ }^{7}$ As shown in [6], a state-space model of the network can be directly obtained from the approach used to derive the network's transfer function.
}

from (8). We start by defining for any set $I \subseteq \mathcal{M}$, a set of input ports $\mathcal{R}_{\mathcal{M} \backslash I}$, from all actuators in $\overline{\mathcal{M}} \backslash I$ - i.e., $\mathcal{R}_{\mathcal{M} \backslash I}=\left\{k \mid \exists i \in \mathcal{M} \backslash I, t_{i k}=1\right\}$.

To show that the new system $\tilde{\Sigma}$ from (11) has no fixed modes with respect to the feedback structure, our goal is to show that for all unstable eigenvalues $\lambda$ of the matrices $\mathbf{A}$ and $\mathbf{A}^{\text {net}}$, we have that $\operatorname{rank}\left(\tilde{\mathbf{M}}_{I, J}(\lambda)\right) \geq n+N$, where

$$
\tilde{\mathbf{M}}_{I, \tilde{J}}(\lambda) \triangleq\left[\begin{array}{ccc}
\mathbf{A}-\lambda \mathbf{I} & \mathbf{0} & \mathbf{B}_{I} \\
\mathbf{B}^{n e t} \mathbf{C} & \mathbf{A}^{n e t}-\lambda \mathbf{I} & \mathbf{0} \\
\mathbf{E}_{\tilde{J}} \mathbf{D}^{n e t} \mathbf{C} & \mathbf{E}_{\tilde{J}} \mathbf{C}^{n e t} & \mathbf{0}
\end{array}\right] .
$$

In the above equation, $\mathbf{E}_{\tilde{J}}$ selects the values from the input ports in network nodes that correspond to the actuators with indices from the set $\mathcal{M} \backslash I$, i.e., $\tilde{J}=\mathcal{R}_{\mathcal{M} \backslash I}$. This is achieved by $\mathbf{E}_{\tilde{J}}$ having a single 1 in each row to select the appropriate values. Now, we can formulate the following lemma.

Lemma 1: An eigenvalue $\lambda$ of $\mathbf{A}$, which is not an eigenvalue of the matrix $\mathbf{A}^{\text {net }}$, is a fixed mode of $\tilde{\Sigma}=\{\tilde{\mathbf{A}}, \tilde{\mathbf{B}}, \tilde{\mathbf{C}}\}$ if and only if it is a fixed mode of the system $\tilde{\Sigma}_{n e w}=$ $\left(\mathbf{A}, \mathbf{B},\left(\mathbf{C}^{n e t}\left(\lambda \mathbf{I}-\mathbf{A}^{n e t}\right)^{-1} \mathbf{B}^{n e t}+\mathbf{D}^{n e t}\right) \mathbf{C}\right)$.

Proof: Consider an eigenvalue $\lambda$ of $\mathbf{A}$ such that $\lambda$ is not an eigenvalue of $\mathbf{A}^{\text {net }}$. Then, $\lambda$ is not a fixed mode of the system $\tilde{\Sigma}=\{\tilde{\mathbf{A}}, \tilde{\mathbf{B}}, \tilde{\mathbf{C}}\}$ if and only if for each $I \subseteq \mathcal{M}$ we have that $\operatorname{rank}\left(\tilde{\mathbf{M}}_{I, \tilde{J}}(\lambda)\right) \geq n+N$. Since $\lambda$ is not an eigenvalue of $\mathbf{A}^{\text {net }}$ it follows that

$$
\begin{aligned}
& \operatorname{rank}\left(\tilde{\mathbf{M}}_{I, \tilde{J}}(\lambda)\right)= \\
& \operatorname{rank}\left[\begin{array}{ccc}
\mathbf{A}-\lambda \mathbf{I} & \mathbf{0} & \mathbf{B}_{I} \\
\mathbf{0} & \mathbf{A}^{n e t}-\lambda \mathbf{I} & \mathbf{0} \\
\mathbf{E}_{\tilde{J}}\left(\mathbf{D}^{n e t}+\mathbf{C}^{n e t}\left(\lambda \mathbf{I}-\mathbf{A}^{n e t}\right)^{-1} \mathbf{B}^{n e t}\right) \mathbf{C} & \mathbf{0} & \mathbf{0}
\end{array}\right] \\
& =N+\operatorname{rank}\left[\begin{array}{ccc}
\mathbf{A}-\lambda \mathbf{I} & \mathbf{B}_{I} \\
\mathbf{E}_{\tilde{J}}\left(\mathbf{D}^{n e t}+\mathbf{C}^{n e t}\left(\lambda \mathbf{I}-\mathbf{A}^{n e t}\right)^{-1} \mathbf{B}^{n e t}\right) \mathbf{C} & \mathbf{0}
\end{array}\right] .
\end{aligned}
$$

Thus, $\operatorname{rank}\left(\tilde{\mathbf{M}}_{I, \tilde{J}}(\lambda)\right)<n+N$ if and only if

$$
\operatorname{rank} \underbrace{\left[\begin{array}{cc}
\mathbf{A}-\lambda \mathbf{I} & \mathbf{B}_{I} \\
\mathbf{E}_{\tilde{J}}\left(\mathbf{D}^{n e t}+\mathbf{C}^{n e t}\left(\lambda \mathbf{I}-\mathbf{A}^{n e t}\right)^{-1} \mathbf{B}^{n e t}\right) \mathbf{C} & \mathbf{0}
\end{array}\right]}_{\tilde{\mathbf{Q}}_{I, \tilde{J}}(\lambda)}<n,
$$

meaning that $\lambda$ is a fixed mode of $\tilde{\Sigma}=\{\tilde{\mathbf{A}}, \tilde{\mathbf{B}}, \tilde{\mathbf{C}}\}$ with respect to feedback structure $\mathbf{T}$ if and only if it is a fixed mode of the system $\tilde{\Sigma}_{n e w}=$ $\left\{\mathbf{A}, \mathbf{B},\left(\mathbf{D}^{\text {net }}+\mathbf{C}^{n e t}\left(\lambda \mathbf{I}-\mathbf{A}^{n e t}\right)^{-1} \mathbf{B}^{n e t}\right) \mathbf{C}\right\}$ with respect to the structural feedback constraints $\mathbf{T}$.

Now, we can proceed to the proof of Theorem 1 .

Proof: [Proof of Theorem 1] Consider any unstable eigenvalue $\lambda$ of the matrix $\mathbf{A}$ that is not an eigenvalue of $\mathbf{A}^{\text {net }}$. In addition, consider any set $I \subseteq \mathcal{M}$ and let $d_{I} \geq 0$ denote the value such that $\operatorname{rank}\left[\begin{array}{ll}\mathbf{A}-\lambda \mathbf{I} & \mathbf{B}_{I}\end{array}\right]=n-d_{I}$. If $d_{I}=0$, we have that $\operatorname{rank}\left(\tilde{\mathbf{Q}}_{I, \tilde{J}}(\lambda)\right) \geq n$, meaning that $\lambda$ is not a fixed mode of the system $\tilde{\Sigma}$.

Since the initial system $\Sigma$ is detectable then $\operatorname{rank}[\underset{\mathbf{C}}{\mathbf{A}-\lambda \mathbf{I}}]=n$ for each unstable eigenvalue $\lambda$ of A [18]. Therefore, if $d_{I}>0$ there are at least $d_{I}$ rows in the matrix $\left[\begin{array}{ll}\mathbf{C} & \mathbf{0}\end{array}\right]$ that are linearly independent of the rows in $\left[\begin{array}{ll}\mathbf{A}-\lambda \mathbf{I} & \mathbf{B}_{I}\end{array}\right]$. With $F_{1}, F_{2}, \ldots, F_{l}$ we represent all possible sets of $d_{I}$ rows of $\mathbf{C}$ that satisfy the above property of linear independence, while $\mathcal{S}_{1}, \mathcal{S}_{2}, \ldots, \mathcal{S}_{l}$ are the sets 
of $d_{I}$ outputs of the plant (i.e., sensors) corresponding to those rows. Consequently, to show that in this case $\operatorname{rank}\left(\tilde{\mathbf{Q}}_{I, \tilde{J}}(\lambda)\right) \geq n$ it is enough to show that there exists $q \in\{1, \ldots, l\}$ such that the row space of $\mathbf{C}_{F_{q}}$ is contained in the row space of $\mathbf{E}_{\tilde{J}}\left(\mathbf{D}^{n e t}+\mathbf{C}^{n e t}\left(\lambda \mathbf{I}-\mathbf{A}^{n e t}\right)^{-1} \mathbf{B}^{n e t}\right) \mathbf{C}$.

Note that $\mathbf{E}_{\tilde{J}}\left(\mathbf{D}_{F_{q}}^{\text {net }}+\mathbf{C}^{\text {net }}\left(\lambda \mathbf{I}-\mathbf{A}^{\text {net }}\right)^{-1} \mathbf{B}_{F_{q}}^{\text {net }}\right)$, where $\mathbf{B}_{F_{q}}^{\text {net }}$ and $\mathbf{D}_{F_{q}}^{\text {net }}$ contain the columns of matrices $\mathbf{B}^{\text {net }}$ and $\mathbf{D}^{\text {net }}$ with indices in $F_{q}$, is effectively $\mathbf{G}_{\operatorname{In}\left(\mathcal{S}_{F_{q}}\right), \operatorname{In}\left(\mathcal{A}_{\mathcal{M} \backslash I}\right)}(\lambda)$ - i.e., the transfer function of the network between the input ports of the sensors with indices in $F_{q}$ and input ports of all actuators with indices in $\mathcal{M} \backslash \mathcal{I}$, evaluated at $\lambda$. Thus, if

$$
\operatorname{rank}\left(\mathbf{G}_{I n\left(\mathcal{S}_{F_{q}}\right), \operatorname{In}\left(\mathcal{A}_{\mathcal{M} \backslash I}\right)}(\lambda)\right)=d_{I},
$$

then the row space of $\mathbf{C}_{F_{q}}$ is contained in the row space of $\mathbf{E}_{\tilde{J}}\left(\mathbf{D}^{n e t}+\mathbf{C}^{n e t}\left(\lambda \mathbf{I}-\mathbf{A}^{\text {net }}\right)^{-1} \mathbf{B}^{n e t}\right) \mathbf{C}_{F_{q}}$. Then using the same approach as in Lemma 1.48 in [16] or Lemma 3 in [3] it can be shown that for almost any choice ${ }^{8}$ of the free parameters from $\Gamma, \operatorname{rank}\left(\tilde{\mathbf{Q}}_{I, \tilde{J}}(\lambda)\right) \geq n$, and furthermore, if

$$
\max _{\Gamma} \operatorname{rank}\left(\mathbf{G}_{I n\left(\mathcal{S}_{F_{q}}\right), \operatorname{In}\left(\mathcal{A}_{\mathcal{M} \backslash I}\right)}(z)\right)=d_{I},
$$

then $\operatorname{rank}\left(\mathbf{G}_{\operatorname{In}\left(\mathcal{S}_{F_{q}}\right), \operatorname{In}\left(\mathcal{A}_{\mathcal{M} \backslash I}\right)}(\lambda)\right)=d_{I}$. Therefore, since the set of parameters for which $\mathbf{A}^{\text {net }}$ is a Schur matrix has measure greater than zero, there exist values for parameters in $\Gamma$ for which $\mathbf{A}^{\text {net }}$ is stable and $\operatorname{rank}\left(\mathbf{G}_{\operatorname{In}\left(\mathcal{S}_{F_{q}}\right), \operatorname{In}\left(\mathcal{A}_{\mathcal{M} \backslash I}\right)}(\lambda)\right)=$ $d_{I}$ for any unstable eigenvalue $\lambda$ of $\mathbf{A}$.

Consequently, since for these network parameters no unstable eigenvalues of $\mathbf{A}$ are eigenvalues of $\mathbf{A}^{\text {net }}$, from Lemma 1 no unstable eigenvalues of $\mathbf{A}$ are fixed modes of the system $\tilde{\Sigma}=\{\tilde{\mathbf{A}}, \tilde{\mathbf{B}}, \tilde{\mathbf{C}}\}$. This implies (from Theorem 2) that there exists a set of controllers at the actuators that can stabilize the system, which concludes the proof.

\section{CONCLUSION}

In this paper we considered the problem of system stabilization using a set of decentralized controllers located at the actuators and a communication network transferring data from the plant's sensors to the controllers. We have shown that when the goal is not to transmit all of the sensors' measurements to every controller but rather to stabilize the system over the network, the capacity constraints of the network become less strict. Specifically, we derived a set of algebraic and topological conditions for the network to satisfy to ensure stabilizability of the plant. These conditions, formulated as min-cut network requirements, depend on the dynamics of the plant (i.e., the maximal geometric multiplicity of any unstable eigenvalue of the plant).

We utilized an algebraic approach to network coding to obtain a description of the network as an LTI system. The key insight in our work is that this model of the network allows for the joint analysis of the network and the plant as a single new dynamical system. Part of our future work will be to generalize our analysis for the case where the network and the plant operate on different sampling rates. Finally, it is worth noting here that the presented approach could also facilitate different types of analysis for NCS. For example,

\footnotetext{
${ }^{8}$ The phrase 'almost any' is used to indicate for all parameters except those from a set of measure zero.
}

a potential avenue for future work is to incorporate security mechanisms from [19], [20] to design intrusion detection schemes capable of detecting attacks on plant actuators and sensors (beside attacks on network nodes).

\section{REFERENCES}

[1] J. P. Hespanha, P. Naghshtabrizi, and Y. Xu, "A survey of recent results in networked control systems," Proceedings of the IEEE, vol. 95, no. 1, pp. 138-162, 2007.

[2] J. Baillieul and P. Antsaklis, "Control and communication challenges in networked real-time systems," Proceedings of the IEEE, vol. 95, no. 1, pp. 9-28, 2007.

[3] M. Pajic, G. J. Pappas, R. Mangharam, and S. Sundaram, "Topological conditions for in-network stabilization of dynamical systems," IEEE J. Sel. Areas Commun., vol. 31, no. 4, pp. 794-807, 2013.

[4] M. Pajic, S. Sundaram, R. Mangharam, and G. J. Pappas, "The Wireless Control Network: A New Approach for Control over Networks," IEEE Trans. Autom. Control, vol. 56, no. 10, pp. 2305-2318, 2011.

[5] S. Yuksel, "Decentralized computation and communication in stabilization of distributed control systems," in Proc. Information Theory and Applications Workshop, ITA, vol. 9, 2009.

[6] M. Kim and M. Médard, "Algebraic network coding approach to deterministic wireless relay networks," arXiv:1001.4431v2 [cs.IT], Jun 2010.

[7] A. Avestimehr, S. Diggavi, and D. Tse, "Wireless network information flow: A deterministic approach," IEEE Trans. Inf. Theory, vol. 57, no. 4, pp. 1872-1905, 2011.

[8] R. Koetter and M. Medard, "An algebraic approach to network coding," IEEE/ACM Trans. Netw., vol. 11, no. 5, pp. $782-$ 795, 2003.

[9] S. Y. Park and A. Sahai, "An algebraic mincut-maxflow theorem," in 2011 IEEE International Symposium on Information Theory Proceedings (ISIT), 2011, pp. 608-612.

[10] — - "Network coding meets decentralized control: Capacitystabilizabililty equivalence," in Proc. 50th IEEE Conference on Decision and Control, 2011, pp. 4817-4822.

[11] S.-H. Wang and E. Davison, "On the stabilization of decentralized control systems," IEEE Trans. Autom. Control, vol. 18, no. 5, pp. 473-478, 1973.

[12] V. Pichai, M. E. Sezer, and D. D. Siljak, "A graph-theoretic characterization of structurally fixed modes," Automatica, vol. 20, no. 2, pp. 247-250, 1984.

[13] C. L. Robinson and P. R. Kumar, "Optimizing controller location in networked control systems with packet drops," IEEE J. Sel. Areas Commun., no. 4, pp. 661-671, 2008.

[14] A. Amaudruz and C. Fragouli, "Combinatorial algorithms for wireless information flow," in Proc. 20th Annual ACM-SIAM Symposium on Discrete Algorithms, 2009, pp. 555-564.

[15] K. J. Reinschke, Multivariable Control A Graph-Theoretic Approach. Springer-Verlag, 1988.

[16] D. Siljak, Decentralized control of complex systems. Academic Press, Inc, 1991.

[17] J. Lavaei and S. Sojoudi, "Time complexity of decentralized fixed-mode verification,” IEEE Trans. Autom. Control, vol. 55, no. 4, pp. 971-976, 2010.

[18] C. Chen, Linear Systems, Theory and Design. Oxford University Press, 1999.

[19] S. Jaggi, M. Langberg, S. Katti, T. Ho, D. Katabi, M. Medard, and M. Effros, "Resilient Network Coding in the Presence of Byzantine Adversaries," IEEE Trans. Inf. Theory, vol. 54, no. 6, pp. 2596-2603, 2008.

[20] S. Sundaram, M. Pajic, C. Hadjicostis, R. Mangharam, and G. J. Pappas, "The Wireless Control Network: Monitoring for Malicious Behavior," in Proc. 49th IEEE Conference on Decision and Control, 2010, pp. 5979-5984. 